\title{
Resuscitation procedures as multi-party dialogue
}

\author{
Ernisa Marzuki, Chris Cummins, \\ Hannah Rohde, Holly Branigan \\ School of Philosophy, Psychology, \\ $\&$ Language Sciences \\ University of Edinburgh \\ Edinburgh, UK \\ s1573599@sms.ed.ac.edu \\ ccummins@exseed.ed.ac.uk \\ hannah.rohde@ed.ac.uk \\ holly.braniganded.ac.uk
}

\section{Abstract}

Successful out-of-hospital cardiac arrest (OHCA) resuscitation relies upon effective team communication, which is evaluated as an aspect of non-technical skills. However, this communication has been largely neglected from a dialogue perspective. We propose addressing this issue by examining the structure of OHCA interaction and its characteristic dialogue features. We explore how speakers verbally signal and align their current states, and the possible trade-off between directness and politeness. Preliminary data suggests frequent use of Assertions in OHCA communication, as in other medical interactions, but that OHCA situations also involve distinctively high proportions of Action-directives. Current states are mostly signalled using explicit State-awareness utterances. Directives' force is also mitigated by politeness features. We discuss how these findings advance our aim of understanding effective team communication in the OHCA context, and how future work might identify associations between linguistic behaviours and resuscitation outcomes.

\section{Introduction}

In modelling the communication structure in dialogue, one productive approach has been to build models of interaction based on annotated dialogue corpora. Using information annotated from reallife interactions, researchers have been able to identify features that are linked to elements such as speaker intention and dialogue outcomes. For example, a corpus of phone conversations was used to develop probabilistic models for predicting call

\author{
Gareth Clegg \\ Resuscitation Research Group \\ University of Edinburgh \\ Department of Emergency Medicine \\ Royal Infirmary of Edinburgh \\ Edinburgh, UK \\ gareth.clegg@ed.ac.uk
}

outcomes and durations (Horvitz and Paek, 2007). Similarly, recorded interactions in a bar were used to derive hypotheses about human interactional behaviours (Loth et al., 2013). In both cases, dialogues were abstracted into models depicting the stages and potential branches of the interaction. The findings were then used to inform interactive systems, helping to establish, in the case of the phone conversations, when to transfer calls from an automated dialogue system to human counterparts and, in the case of the bar scenes, how a robot bartender might identify speakers' signals of their intention to place an order for drinks.

The present study applies a similar approach to a category of interactions in the medical domain: out-of-hospital cardiac arrest (OHCA) resuscitations. From a dialogue perspective, this represents a case study of a high-stakes, time-constrained team interaction, allowing us to explore the usefulness of dialogue modelling for this domain. From a medical perspective, it represents an attempt to use dialogue modelling to better understand and potentially enhance communication between medical experts when they work as a team.

Existing work related to dialogue modelling in the medical realm primarily focuses on expertnon-expert interactions (Ford et al., 2000; Laws et al., 2011; McNeilis, 1995; Roter and Larson, 2001; Stiles, 1978). Such studies provide insight into inter-medical communication, but they say little about the intra-medical domain. Medical team communication in high-stakes contexts, like surgery and resuscitation, has been understudied from the perspective of dialogue research. Within the medical community, the training and evaluation of team communication has largely eschewed theoretical linguistic input, instead focusing on the subjective judgment of team communication as part of the evaluation of non-technical skills (NTS). 